\title{
Flux decay during thermonuclear X-ray bursts analysed with the dynamic power-law index method
}

\author{
J. Kuuttila ${ }^{1}$, J. J. E. Kajava ${ }^{2,1,3}$, J. Nättilä ${ }^{1,4}$, S. E. Motta ${ }^{5}$, C. Sánchez-Fernández ${ }^{3}$, E. Kuulkers ${ }^{3,6}$, \\ A. Cumming ${ }^{7}$, and J. Poutanen $1,4,8$ \\ 1 Tuorla Observatory, Department of Physics and Astronomy, University of Turku, Väisäläntie 20, 21500 Piikkiö, Finland \\ e-mail: jmjkuu@utu.fi \\ 2 Finnish Centre for Astronomy with ESO (FINCA), University of Turku, Väisäläntie 20, 21500 Piikkiö, Finland \\ ${ }^{3}$ European Space Astronomy Centre (ESA/ESAC), Science Operations Department, 28691 Villanueva de la Cañada, Madrid, Spain \\ 4 Nordita, KTH Royal Institute of Technology and Stockholm University, Roslagstullsbacken 23, 10691 Stockholm, Sweden \\ 5 University of Oxford, Department of Physics, Astrophysics, Denys Wilkinson Building, Keble Road, Oxford OX1 3RH, UK \\ 6 ESA/ESTEC, Keplerlaan 1, 2201 AZ Noordwijk, The Netherlands \\ 7 Department of Physics and McGill Space Institute, McGill University, 3550 University Street, Montreal, QC H3A2T8, Canada \\ 8 Kavli Institute for Theoretical Physics, University of California, Santa Barbara, CA 93106, USA
}

Received 20 March 2017 / Accepted 9 May 2017

\begin{abstract}
The cooling of type-I X-ray bursts can be used to probe the nuclear burning conditions in neutron star envelopes. The flux decay of the bursts has been traditionally modelled with an exponential, even if theoretical considerations predict power-law-like decays. We have analysed a total of 540 type-I X-ray bursts from five low-mass X-ray binaries observed with the Rossi X-ray Timing Explorer. We grouped the bursts according to the source spectral state during which they were observed (hard or soft), flagging those bursts that showed signs of photospheric radius expansion (PRE). The decay phase of all the bursts were then fitted with a dynamic power-law index method. This method provides a new way of probing the chemical composition of the accreted material. Our results show that in the hydrogen-rich sources the power-law decay index is variable during the burst tails and that simple cooling models qualitatively describe the cooling of presumably helium-rich sources 4 U 1728-34 and 3A 1820-303. The cooling in the hydrogen-rich sources 4U 1608-52, 4U 1636-536, and GS 1826-24, instead, is clearly different and depends on the spectral states and whether PRE occurred or not. Especially the hard state bursts behave differently than the models predict, exhibiting a peculiar rise in the cooling index at low burst fluxes, which suggests that the cooling in the tail is much faster than expected. Our results indicate that the drivers of the bursting behaviour are not only the accretion rate and chemical composition of the accreted material, but also the cooling that is somehow linked to the spectral states. The latter suggests that the properties of the burning layers deep in the neutron star envelope might be impacted differently depending on the spectral state.
\end{abstract}

Key words. stars: neutron $-\mathrm{X}$-rays: binaries $-\mathrm{X}$-rays: bursts - accretion, accretion disks

\section{Introduction}

X-ray bursts were first discovered in 1975 by Grindlay et al. (1976), who observed bright flashes coming from a low-mass X-ray binary (LMXB), 3A 1820-303 (but see also Belian et al. 1972; Babushkina et al. 1975). Since then, type-I thermonuclear X-ray bursts have been observed from more than $100 \mathrm{LMXBs}$ (Galloway et al. 2008) ${ }^{1}$. X-ray bursts are triggered by the accreted matter from the companion star that piles up on the neutron star (NS) surface until the pressure is sufficiently high for the ignition of the thermonuclear fusion (Bildsten 1998). The burning is usually unstable and most of the accumulated material burns in a matter of seconds (for a review, see Lewin et al. 1993; Strohmayer \& Bildsten 2006). Some of these bursts are so energetic that they reach the local Eddington limit, causing the atmosphere of the star to momentarily expand during the burning. These are often dubbed as the photospheric radius expansion (PRE) bursts.

\footnotetext{
1 See also http://burst.sci.monash.edu/sources and https: //personal.sron.nl/ jeanz/bursterlist.html
}

The bursting behaviour may be affected significantly by the spectral (or accretion) state of the binary (see e.g. Galloway et al. 2008; Kajava et al. 2014, and references therein). Many bursting NSs in LXMBs alternate between so-called hard and soft spectral states (Muñoz-Darias et al. 2014), and X-ray bursts can occur while the LMXB is in either of those two states or during state transitions (i.e. the intermediate state). In the hard state the energy spectrum is dominated by Comptonised emission (see e.g. Done et al. 2007). The optically thick accretion disc is likely truncated at some distance from the NS, and a hot, optically thin, and geometrically thick inner flow channels the accreted gas onto the NS. In the soft state the accretion disc is thought to reach the surface of the NS and two thermal components dominate the energy spectrum. The accretion disc dominates the X-ray emission in soft X-rays, and a hotter optically thick spreading layer that is thought to form in the NS-accretion disc boundary (Inogamov \& Sunyaev 1999; Suleimanov \& Poutanen 2006; Inogamov \& Sunyaev 2010) emits harder X-rays. Importantly, according to Inogamov \& Sunyaev (2010) a significant fraction of the accreted energy can be dissipated in the burning layers, 
and it is thus possible that the accretion state is a key driver of the bursting properties.

Observationally, type-I X-ray bursts are characterised by a rapid rise of the $X$-ray flux by a factor of up to $\sim 100$ with respect to the persistent emission, after which the X-ray flux slowly decreases back to the levels prior to the burst, usually within a minute or two. The flux decay has traditionally been modelled with exponential functions because early attempts to explain the bursts predicted an exponential decay, and these models matched well the decay of the first bursts observed (Grindlay \& Gursky 1976). On the other hand, a simple physical consideration of one-dimensional radiative heat transfer implies a power-law-like dependency for the observed flux, and in't Zand et al. (2014) showed that the single power law successfully explains the flux decays for a subset of $35 \mathrm{X}$-ray bursts from 14 sources.

In both cases (exponential decays or power-law models) the decay rate was assumed to be constant throughout the cooling tail (in't Zand et al. 2014). However, cooling models developed by Cumming \& Macbeth (2004) predict that the powerlaw decay index should decrease while the flux drops. These models were initially constructed for superbursts, and later in't Zand et al. (2014) adapted these models for shallower column depths with an energy release of $1.6 \mathrm{MeV}$ per nucleon, as expected from complete helium burning to evaluate the behaviour of normal bursts. These models assume an instantaneous energy injection and then follow the subsequent thermal evolution of the burning layer. They predict a smooth decrease of the power-law index, from over 2 towards 1 (or even lower depending on the parameters) with decreasing flux (see Fig. 6 of in't Zand et al. 2014). Thus, it is clear that the full cooling behaviour cannot be fully described with only one or two power laws fitted to the burst tail nor is an exponential decay model sufficient to capture all of the relevant physics.

In this paper we study how the predicted decay rate changes during type-I X-ray bursts of five LMXBs using a dynamic power-law index method, where a power law is fitted to the fluxtime evolution within a moving time window. As a first step of this kind of dynamic decay modelling, we compare the observations to the aforementioned simple models developed in Cumming \& Macbeth (2004) and in in't Zand et al. (2014). The five systems have hydrogen-rich and hydrogen-poor accretors, which also allows us to study how the chemical composition affects details of the cooling. In addition, we study the burst decay behaviour as a function of spectral state to see if the accretion geometry affects the conditions in the burning depths. Finally, we investigate whether the Eddington limited PRE bursts have different cooling behaviour compared to the fainter non-PRE bursts.

\section{Observations}

\subsection{The data}

We obtained all available public Rossi X-ray Timing Explorer/Proportional Counter Array (RXTE/PCA) data from the High Energy Astrophysics Science Archive Research Center ${ }^{2}$ (HEASARC) for the sources 4U 1636-536, 4U 1608-52, and $4 \mathrm{U} 1728-34$. We selected these sources because of the large number of bursts observed from them by RXTE (about 300 from $4 \mathrm{U}$ 1636-536, 120 from 4U 1728-34, and 50 from 4U 160852) and because they show both PRE and non-PRE bursts in both the hard and soft states, which allows us to investigate the

2 http://heasarc.gsfc.nava.gov effect of the spectral state on the X-ray burst decay rates. We included also 3A 1820-303 (=4U 1820-30) in this work, because it is a well-known ultra-compact binary accreting heliumrich material from its companion (Stella et al. 1987), which produces only photospheric expansion bursts in the hard state (García et al. 2013). Thus, this interesting comparison source helps us to understand the effects of spectral state and chemical composition on the cooling rates. In addition, we included GS 1826-24 because of its very regular bursting behaviour and known hydrogen-rich composition of the accreted material (Galloway et al. 2004).

The X-ray burst spectral data were already used in Poutanen et al. (2014) and Kajava et al. (2014), where the data reduction method is described in detail. As in those papers, we extracted time-resolved spectra using initial time resolution of $0.25,0.5,1.0$, or $2.0 \mathrm{~s}$ based on the peak count rate $(>6000$, $6000-3000$, 3000-1500, or $<1500$ count per second, respectively). Then we doubled the time resolution every time the count rate decreased by a factor of $\sqrt{2}$ to maintain the same signalto-noise ratio. We modelled the burst emission using a blackbody spectrum (BBODYRAD model in XSPEC) modified by interstellar absorption (TBABS). We fixed the absorption column density $N_{\mathrm{H}}$ to constant values $0.16 \times 10^{22} \mathrm{~cm}^{-2}$ for $3 \mathrm{~A} 1820$ 303 (Kuulkers et al. 2003), $0.40 \times 10^{22} \mathrm{~cm}^{-2}$ for GS 182624 (in't Zand et al. 1999), $2.60 \times 10^{22} \mathrm{~cm}^{-2}$ for 4 U 1728-34 (D'Aí et al. 2006), $0.25 \times 10^{22} \mathrm{~cm}^{-2}$ for 4U 1636-536 (Asai et al. 2000), and $0.89 \times 10^{22} \mathrm{~cm}^{-2}$ for $4 \mathrm{U} 1608-52$ (Keek et al. 2008). The best-fitting parameters were then the blackbody temperature $T_{\mathrm{bb}}$ and the normalisation constant $K_{\mathrm{bb}}=\left(R_{\mathrm{bb}}[\mathrm{km}] / d_{10}\right)^{2}$, where $d_{10} \equiv d / 10 \mathrm{kpc}$ is the distance in units of $10 \mathrm{kpc}$. The blackbody flux $F_{\text {bb }}$ was computed using the CFLUX convolution model over the $0.01-100 \mathrm{keV}$ band, and the parameter errors were calculated as $1 \sigma$ confidence levels. We adopted the same criteria described in Galloway et al. (2008) to determine if a burst showed signs of PRE, i.e. photospheric radius expansion was considered to occur when the blackbody temperature evolution showed a characteristic double-peaked structure and the blackbody normalisation reached its maximum at the same time as the temperature was at its minimum.

Following Poutanen et al. (2014) and Kajava et al. (2014), we extracted a 16-s spectrum prior to the burst and then subtracted it as a background for each burst (Kuulkers et al. 2002; Galloway et al. 2008). While this standard approach is not completely accurate, as the persistent flux might change during the bursts (see e.g. van Paradijs \& Lewin 1986; Sztajno et al. 1986; Kuulkers et al. 2002; Worpel et al. 2013), such flux variations are not expected to be as significant in the cooling tails as during the other phases of the burst (see Figs. 2 and 6 of Worpel et al. 2013). Therefore, this operation does not significantly affect the results of our analysis.

\subsection{Accretion states}

The different accretion states of LMXBs show distinct patterns of spectral and timing behaviour (Hasinger \& van der Klis 1989). Thus the states can be classified based on both the X-ray hardness (e.g. Kajava et al. 2014) and the total fractional rms of the variability (Muñoz-Darias et al. 2011).

Muñoz-Darias et al. (2014) defined three main spectral states in accreting NS X-ray binaries, based on the total fractional rms: a hard state at rms $>20 \%$, an intermediate state at rms between 5 and $20 \% \mathrm{rms}$, and a soft state at rms $<5 \%$. However, these authors note that different sources might span different rms ranges 
and the state classification given above has to be adjusted. On the other hand, X-ray colours have been historically used to determine the spectral states of accreting NS, but while in certain sources the distinction between hard and soft states (i.e. roughly corresponding to the so-called island state and the banana state of Hasinger \& van der Klis 1989) is clear, in others it remains somewhat arbitrary (Kajava et al. 2014).

In order to quantify a possible dependence of the burst flux decay on the spectral state, we classified the bursts of our sample into two different groups based on the spectral state during which they were observed. As suggested by Hasinger \& van der Klis (1989), the least ambiguous way to determine the spectral state is using both the rms and colour information (see Fig. 1). We obtained X-ray colours and persistent fluxes following Kajava et al. (2014) in the 3-4 to 4-6.4 keV energy band (soft colour) and 6.4-9.7 to $9.7-16 \mathrm{keV}$ energy band (hard colour). We measured the rms following Muñoz-Darias et al. (2014) in the $2-16 \mathrm{keV}$ energy band and in the $0.1-64 \mathrm{~Hz}$ frequency band. Both the colours and rms were measured from the persistent emission before the onset of each burst.

Comparing the state classification defined by Muñoz-Darias et al. (2014) and the X-ray colour analysis reported by Kajava et al. (2014) for 4U 1636-536, 4U 1608-52, and 4U 1728-34, we found that for a few bursts the two methods resulted in ambiguous classifications (particularly for 4U 1636-536), and in those cases we adjusted the limits so that both colour and rms diagrams had the least ambiguities. We also find that (see Fig. 1)

- Most of the bursts found at rms lower than 9\% are produced in the soft state. We refer to these bursts as soft bursts.

- The majority of the bursts observed above $9 \%$ rms are generally found in either the intermediate or hard state. We generically refer to these bursts as hard bursts.

\section{Analysis}

\subsection{Theory}

It has been customary to model the cooling phase of an X-ray burst with a simple exponential decay (see e.g. Grindlay \& Gursky 1976; Galloway et al. 2008). While some bursts can be described with exponential decay, it does not fit well all the bursts nor is it based on correct physical considerations. By considering one-dimensional radiative heat transfer and the Stefan-Boltzmann law, in't Zand et al. (2014) showed that the burst luminosity should follow a power-law-like decay

$$
L \propto t^{-\alpha}
$$

where the power-law index $\alpha$ equals $4: 3$ if the dominant contribution to the heat capacity comes from ions, $\alpha=2$ if it is dominated by degenerate electrons, or $\alpha>2$ if the heat capacity is dominated by the radiation field. These cooling regimes are illustrated in Figs. 2c and d. The bursting NS envelope is a mixture of all these components, so the decay index should lie somewhere in between these values. in't Zand et al. (2014) have also mentioned that a similar consideration of simple conductive heat transfer produces an exponential decay, but they have found that the power law was always a better representation of the cooling with majority of the bursts having the $\alpha$ between 4:3 and 2 . On the other hand, they had selected only a small subset of 'clean' and ordinary bursts to get a clear view of the cooling process without the contribution of prolonged nuclear burning, varying accretion rate, or accretion disc. While many bursts indeed follow the power-law decay very well (Fig. 2a), there are also bursts that do not, nor can they be fitted with an exponential decay (Fig. 2b). Models of Cumming \& Macbeth (2004), on the other hand, predict that the cooling rate changes during the burst, i.e. the power-law index is varying, which could be expected if the governing component changes with the temperature and flux.

\subsection{Temporal evolution of the flux}

To study the possible changing cooling rate of X-ray bursts, we present a dynamic power-law index method, where within a moving time window we fit a power-law function of time to the flux as follows:

$F(t) \approx F_{0}\left(t / t_{0}\right)^{-\alpha}$

where $t$ is the time in seconds, $F_{0}$ is a free parameter (whose value is usually close to the flux of the first data point of the window $t_{0}$ ), and $\alpha$ is the power-law decay index ${ }^{3}$

$\alpha=-\frac{\mathrm{d} \log F}{\mathrm{~d} \log t}$

Low values of $\alpha$ correspond to a slow local change in the flux, whereas high values of $\alpha$ indicate a fast change.

We adopted a time window of seven bins and fitted a power law to the measured burst blackbody flux values in this narrow time range, then moved the window one step forward and fitted again and so on until the end of the burst, which we identify when typically a flux $\sim 5 \%$ of the peak flux is reached. We fitted the whole burst, but consider only the cooling part, where the $\alpha$ is positive. The first fits with positive $\alpha$ are still affected by the peak of the burst, which means that at first the $\alpha$-values rise rapidly before settling to the actual decay values.

The effect of the selected window size on the results was also investigated and was found to have no impact on the underlying $\alpha$-evolution, within a reasonable time range, as shown in Figs. 2c and d, which provide $\alpha$ as a function of time for windows of 3, 7, and 11 point fits. However, a narrower window produced noisier results, while larger number of points tends to smooth the $\alpha$ flux evolution too much. As a good balance between these two effects we found that a 7 point fit produces the best result for our data reduction and bin summation methods (see Sect. 2 and also Poutanen et al. 2014; and Kajava et al. 2014).

\section{3. $\alpha$-flux evolution}

In Figs. 3-7 we show the values of the power-law index $\alpha$ obtained from the moving time-window fits as a function of the burst flux. These figures show how $\alpha$, i.e. the cooling rate, changes as the burst flux decays. In these figures the peak of the burst is on the right-hand side and the flux decreases from right to left. The bursts are grouped based on the accretion state and whether or not they are PRE bursts.

In Figs. 3-7 we also show the burst cooling models adapted from in't Zand et al. (2014). These models include their onezone model with an ignition depth of $10^{8} \mathrm{~g} \mathrm{~cm}^{-2}$ and three multizone models with ignition depths of $10^{8}, 10^{9}$, and $10^{10} \mathrm{~g} \mathrm{~cm}^{-2}$, which are based on the work of Cumming \& Macbeth (2004).

\footnotetext{
3 Instead of assuming a separate $\alpha$ value for each bin, we could also try to capture its evolution by assuming some a priori functional form for the function $\alpha(t)$ and try to constrain that using the information from the whole burst tail. However, this kind of formulation does not allow us to examine any unexpected or irregular cooling behaviour that we observe here.
} 

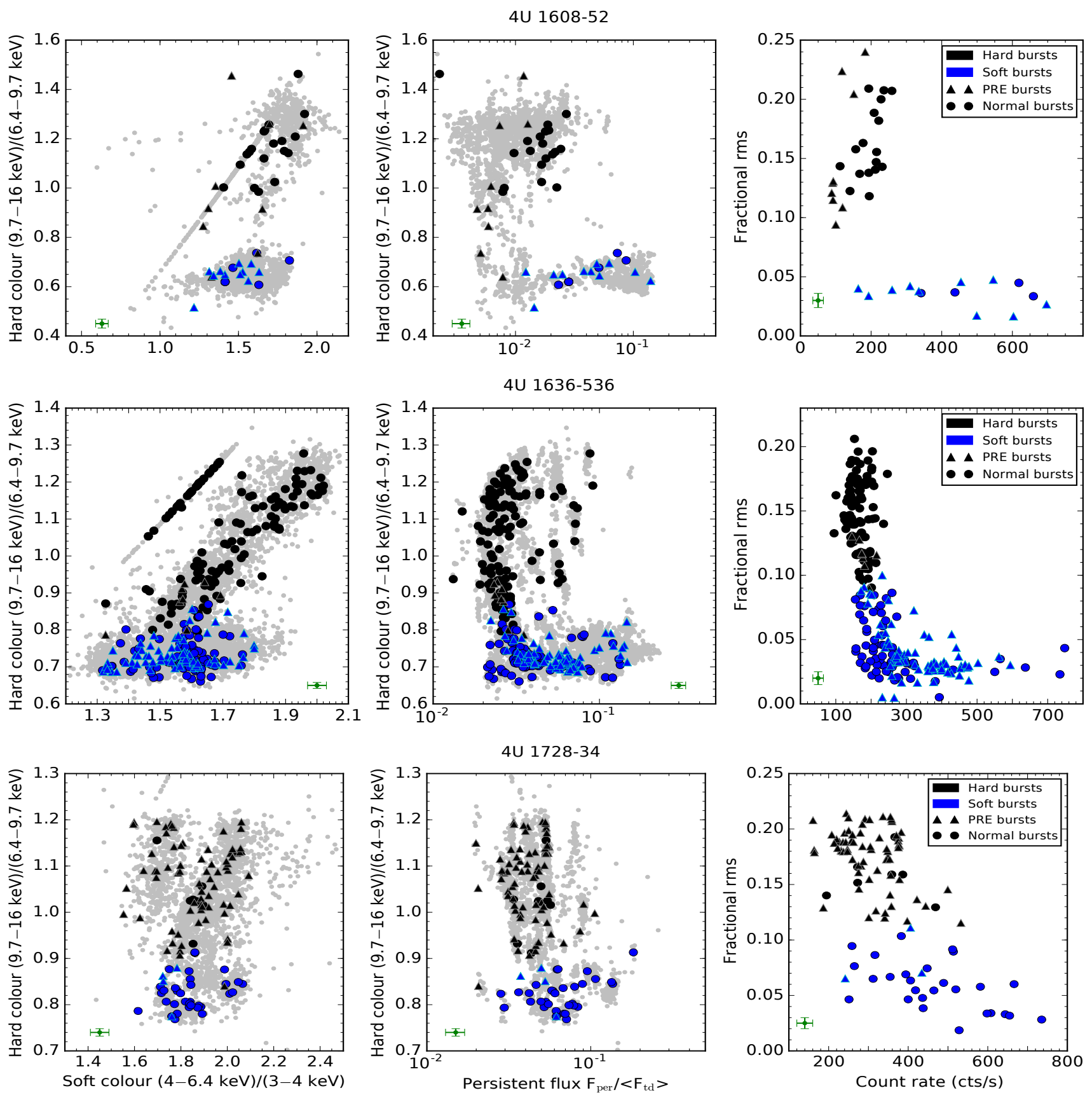

Fig. 1. State separation for $4 \mathrm{U} 1636-536,4 \mathrm{U}$ 1608-52, and 4U 1728-34. The left side panels are the colour-colour diagrams from Kajava et al. (2014) with the soft and hard state bursts indicated with blue and black markers, and PRE bursts and normal bursts marked with triangles and circles, respectively. The diagonal lines in the left panels are due to our energy band selection and the fact that the persistent emission can sometimes be described with a power law (see Kajava et al. 2014, for details). In the middle panels, the persistent flux normalised with the average touchdown flux is on the $x$-axis and the hard colour is on the $y$-axis (see Kajava et al. 2014). The right-hand panels show the dependence of the rms on the count rate used to separate the bursts into two states, as explained in Sect. 2.2 (see also Muñoz-Darias et al. 2014). The green crosses in the bottom of each panel show the typical error bars.

The fluxes provided by these models were arbitrarily scaled to coincide with the fluxes measured for each source. In practice this scaling connects the emergent and observed flux from the source,

$F=\frac{F_{\mathrm{NS}}}{(1+z)^{2}} \frac{R^{2}}{D^{2}}$

where $F$ is the observed flux, $F_{\mathrm{NS}}$ is the flux at the NS surface, $R$ is the NS radius, $1+z=\left(1-2 G M / R c^{2}\right)^{-1 / 2}$ is the redshift, $G$ is the gravitational constant, $M$ is the NS mass, $c$ is the speed of light, and $D$ is the distance to the source. The scaling applied to the models is consistent within a factor of $2-3$ with what one would expect from Eq. (4) for a canonical NS $\left(M=1.4 M_{\odot}, R=10 \mathrm{~km}\right)$ with distances $5.3 \pm 0.8 \mathrm{kpc}$ for $4 \mathrm{U} 1728-34$ (Jonker \& Nelemans 2004), $6.0 \pm 0.5 \mathrm{kpc}$ for $4 \mathrm{U}$ 1636-536 (Galloway et al. 2006), $7.6 \pm 0.4 \mathrm{kpc}$ for $3 \mathrm{~A} 1820-303$ (Heasley et al. 2000), $3.4 \pm 0.3 \mathrm{kpc}$ for 4U 1608-52 (Poutanen et al. 2014), and $5.7 \pm 0.2 \mathrm{kpc}$ for GS 1826-24 (Chenevez et al. 2016). Here the distance to the source is by far the largest origin of error in the scaling. 

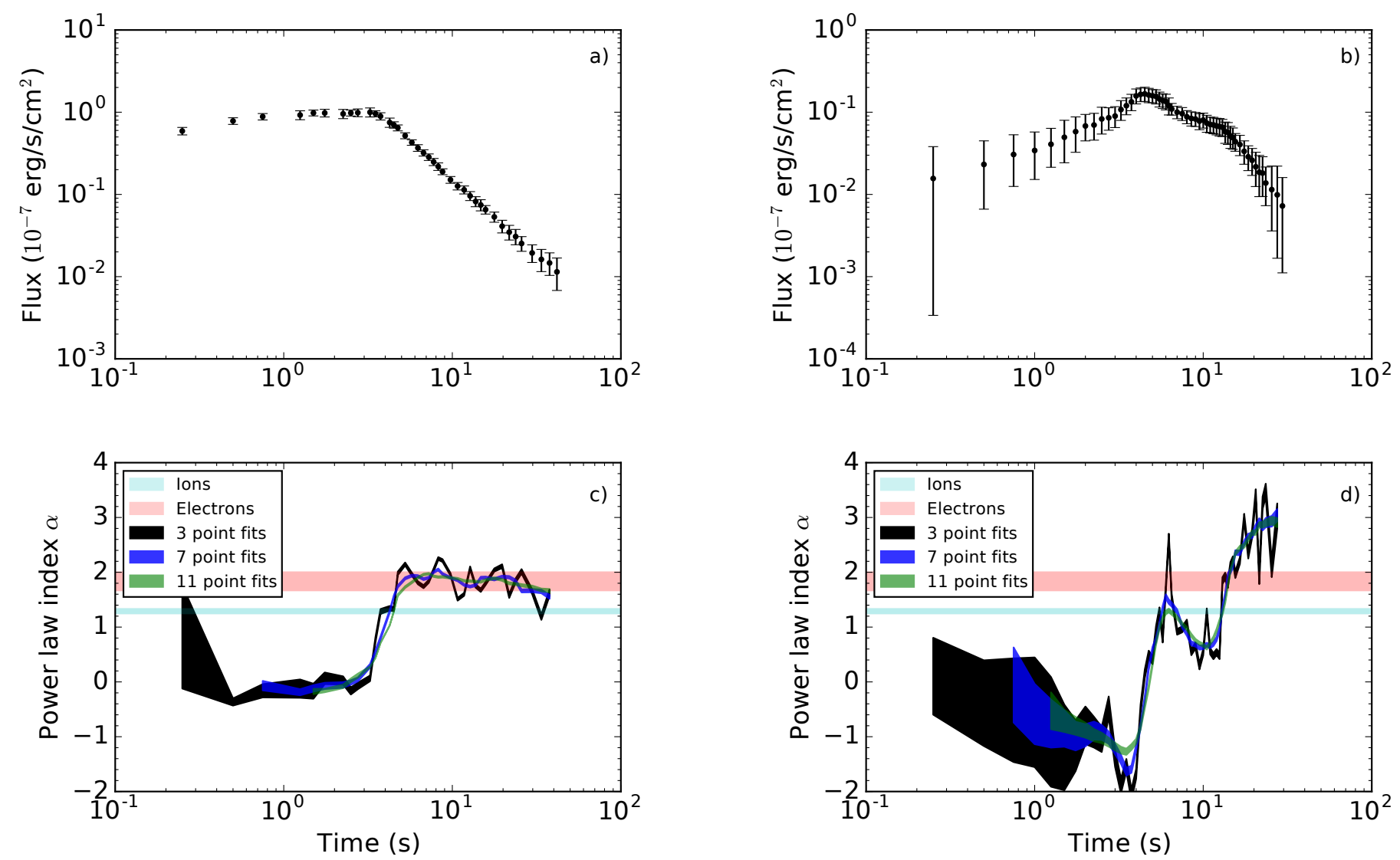

Fig. 2. Two examples of application of the dynamic power-law index method to the bursts. The upper panels show the temporal evolution of the flux, while the lower panels give power-law indices. Left panels: aPRE burst from 4U 1728-34 (MJD 51 238.79155), which shows a nearly power-law dependence of flux on time; the power-law index $\alpha$ varies smoothly (panel $c$ )). Right panels: a non-PRE burst from 4U 1636-536 (MJD 52 290.14847), whose temporal evolution does not follow a power law with the corresponding power-law index shown in panel $d$ ). In the lower panels the black, blue, and green areas represent the $1 \sigma$ confidence levels of the power-law indices of the individual 3, 7, and 11 point fits, respectively. The power-law indices are shown for the entire burst, but we study only the cooling tails. Also in the lower panels the red area shows the typical $\alpha$-regime if the cooling is dominated by electrons, and the cyan area corresponds to ion-dominated cooling (see text).

In the following Sects. 3.3.1-3.3.5 we describe the results of each source in detail. In this work we focus mostly the $\alpha$-flux evolution of the bursts, even though both the flux and $\alpha$ are also functions of time. One should keep in mind that even a small change in $\alpha$-flux evolution can mean a large change in, for example the burst duration.

\subsubsection{A 1820-303}

3A $1820-303$ (=4U 1820-30) (Fig. 3) is an ultra-compact binary with an orbital period of just 11.4 min (Stella et al. 1987). The companion star is a low-mass helium white dwarf, which means that the accreting material and thus the burning material on the surface of the NS, is mainly helium (Rappaport et al. 1987). In 3A 1820-303 all the bursts occur in the hard state and show signs of PRE.

From Fig. 3 we can see that all the bursts from this source follow the same behaviour. After the peak of the burst, $\alpha$ rises quickly to about 2.2 , after which it starts to slowly decrease with the flux being around 2 most of the time. This means that the cooling is consistent with heat transfer dominated by electrons. At low fluxes, $\alpha$ seems to rise again, while at the same time the scatter increases, which is likely due to the burst flux approaching the persistent emission level. There is a qualitative similarity between the models and data, but the models predict lower values of $\alpha$, i.e. slower cooling, than we see in the data, especially at lower fluxes. A similar discrepancy between the cooling rate of models and data is also seen in a superburst from 4U 1636-536 (Keek et al. 2015; but see also Koljonen et al. 2016).

\subsubsection{GS $1826-24$}

GS 1826-24 (also known as Ginga 1826-238) is a very extensively studied burster because of its bright, frequent, and regular bursts. Ubertini et al. (1999) dubbed it the "clocked burster" because of the regular bursting behaviour, while Bildsten (2000) name it the "textbook burster" because of the good agreement with theory.

Bildsten (2000) suggested that the bursts involve mixed $\mathrm{H} / \mathrm{He}$ burning, where an initial helium flash prompts prolonged hydrogen burning via the rp-process (Wallace \& Woosley 1981). This naturally explains the observed long tails $(\approx 100 \mathrm{~s})$ of the bursts (Heger et al. 2007). Galloway et al. (2004) arrived at the same conclusion by showing that the regular bursting behaviour is well understood as being due to He ignition in a H-rich environment. The inferred accretion rate $\dot{M} \approx 10^{-9} M_{\odot} \mathrm{yr}^{-1}$ implies that hydrogen burns also stably between the bursts by the betalimited hot CNO-cycle (Galloway et al. 2004).

The regular and very well understood behaviour makes GS 1826-24 an ideal calibration source for hydrogen rich bursts. In Fig. 4 we show the results of the dynamic power-law method for this source. From this figure we can see that all the bursts 


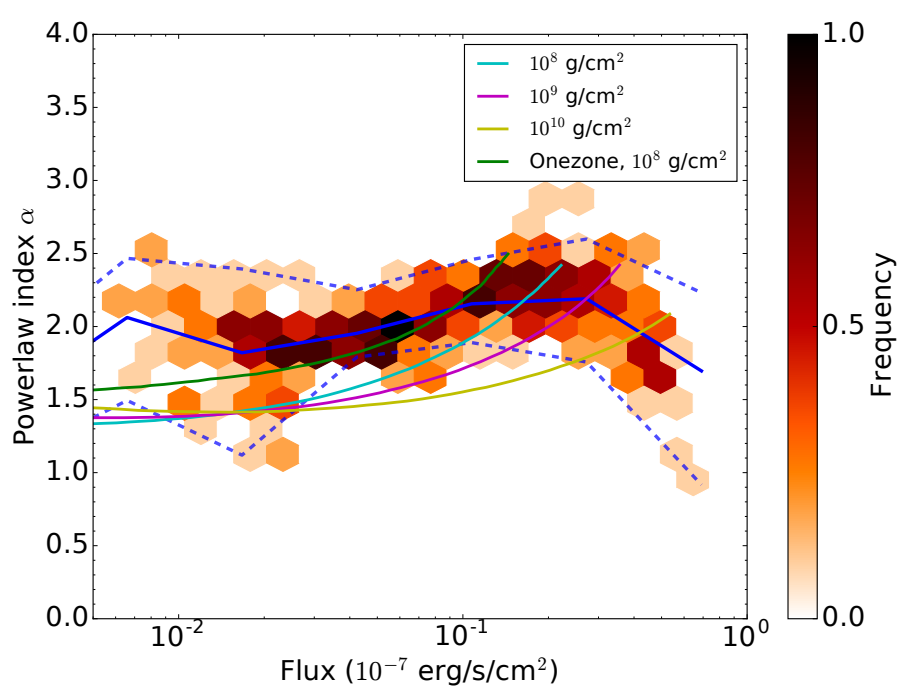

Fig. 3. Dependence of the power-law index $\alpha$ on the burst flux for all bursts from 3A 1820-303 (all of these bursts are hard-state PRE bursts). The data are shown in a two-dimensional histogram, where the colour of a bin shows the frequency of occurrences (normalised to the peak value) according to the colour bar on the right. The solid blue line is the average of the data and the blue dashed lines show the $1 \sigma$ limits. The solid green line shows the one-zone model with a column depth of $10^{8} \mathrm{~g} \mathrm{~cm}^{-2}$, and the cyan, magenta, and yellow lines show the three multi-zone models with ignition depths of $10^{8}, 10^{9}$, and $10^{10} \mathrm{~g} \mathrm{~cm}^{-2}$, respectively, based on the work of Cumming \& Macbeth (2004).

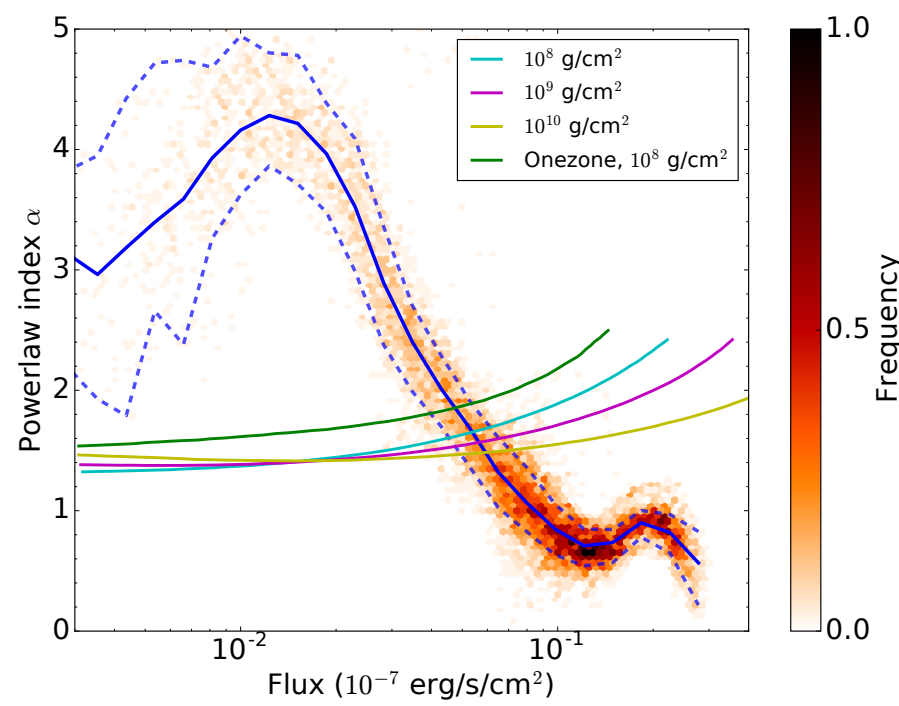

Fig. 4. Same as Fig. 3, but for GS 1826-24.

indeed follow closely the same behaviour, where the $\alpha$ first rises quickly to about 1 , after which it first slightly decreases to around $\alpha \approx 0.8$ and then rises dramatically to all the way to $\alpha \approx 4$. The rise in $\alpha$ is almost linear, which means that the flux decays almost exponentially, since in the $\alpha$ versus flux space an exponential function is a straight inclined line. At low burst fluxes the $\alpha$ decreases again, but the scatter is substantial at the end of the cooling tail.

\subsection{3. $4 U 1728-34$}

$4 \mathrm{U}$ 1728-34 (Fig. 5) is also a H-poor ultra-compact binary candidate (Galloway et al. 2010), and the burst properties indeed closely resemble those of 3A 1820-303 (see e.g.
Galloway et al. 2008). However, unlike 3A 1820-303, 4U 172834 does have bursts both in the hard and soft state and not all of the bursts show a PRE stage. In 4U 1728-34 both the normal and PRE bursts cool down approximately in the same way as in $3 \mathrm{~A}$ 1820-303, regardless of the accretion state. The non-PRE bursts in the soft state just have slightly lower peak fluxes and more burst-to-burst variations because they are fainter. The bursts from this source show a clear similarity to the models with the lowflux decay indices closer to the predicted value of $\alpha \sim 1.4$ than in 3 A $1820-303$.

\subsection{4. $4 \bigcup 1636-536$}

$4 \mathrm{U} 1636-536$ (Fig. 6) is believed to accrete both $\mathrm{H}$ and He because its 18 mag optical counterpart V801 Arae has $3.8 \mathrm{~h}$ orbital period and clear hydrogen emission lines (van Paradijs et al. 1990; Augusteijn et al. 1998; Giles et al. 2002). In this source all the PRE bursts except six happen in the soft state and even these six happen during the transition from one state to the other and they resemble the soft state more than the actual hard state bursts.

All the PRE bursts in this source (lower panels of Fig. 6) closely resemble the bursts from 3A $1820-303$ and 4U 172834 . They also qualitatively follow the models, but there are some bursts with clearly higher and lower $\alpha$-values.

The hard state normal bursts in 4U 1636-536 (upper right panel of Fig. 6) clearly differ from the PRE bursts. The index $\alpha$ first rises quickly when the burst starts to cool and then it starts to decrease slowly as in the PRE bursts. At lower fluxes, however, $\alpha$ starts to rise again reaching values as high as $\alpha \approx 4$, i.e. the bursts cool down much more rapidly than predicted by the cooling models. This behaviour is similar to GS 1826-24, but whereas the $\alpha$ in GS 1826-24 stays below 1 in the beginning of the cooling, in $4 \mathrm{U} 1636-536$ it rises at first close to 2 before stalling and rising again. Overall, the shape of the $\alpha$-flux curve of the hard state normal bursts is close to that of GS 1826-24, but the actual values differ.

The normal bursts that occur in the soft state do not follow the behaviour of either of the two calibration sources. Overall, these bursts lack the decrease of $\alpha$ altogether, seen in the other bursts and sources. The burst flux decay seems to intensify almost monotonically as the atmosphere cools down. Also, the effects in the tail are not due to the background emission subtraction as we carefully tested different data reduction schemes but still obtained the same behaviour.

\subsection{5. $4 \mathrm{U} 1608-52$}

Similar to $4 \mathrm{U}$ 1636-536, 4U 1608-52 (Fig. 7) is also believed to accrete both $\mathrm{H}$ and $\mathrm{He}$, since its optical counterpart QX Nor is likely a late F- or an early G-type main sequence star with an orbital period of $0.537 \mathrm{~d}$ (Wachter et al. 2002). The normal bursts of $4 \mathrm{U} 1608-52$ in the hard state are similar to those of $4 \mathrm{U}$ 1636-536, having the peculiar rise of $\alpha$ at low fluxes, but in this source the $\alpha$ rises only to around 2.5 compared to the $\alpha \approx 4$ in $4 \mathrm{U}$ 1636-536. However, in the soft state normal bursts there does not seem to be any rise in the $\alpha$-values at low fluxes, but instead they nearly monotonically decrease during the decay.

The PRE bursts that occur in the soft state clearly follow the same behaviour as the PRE bursts in 4U 1636-536 and all the bursts in 4U 1728-34 and 3A 1820-303. Unlike in 4U 1636536, 4U 1608-52 has several PRE bursts in the hard state and they are different from the soft state bursts. There is a clear rise 

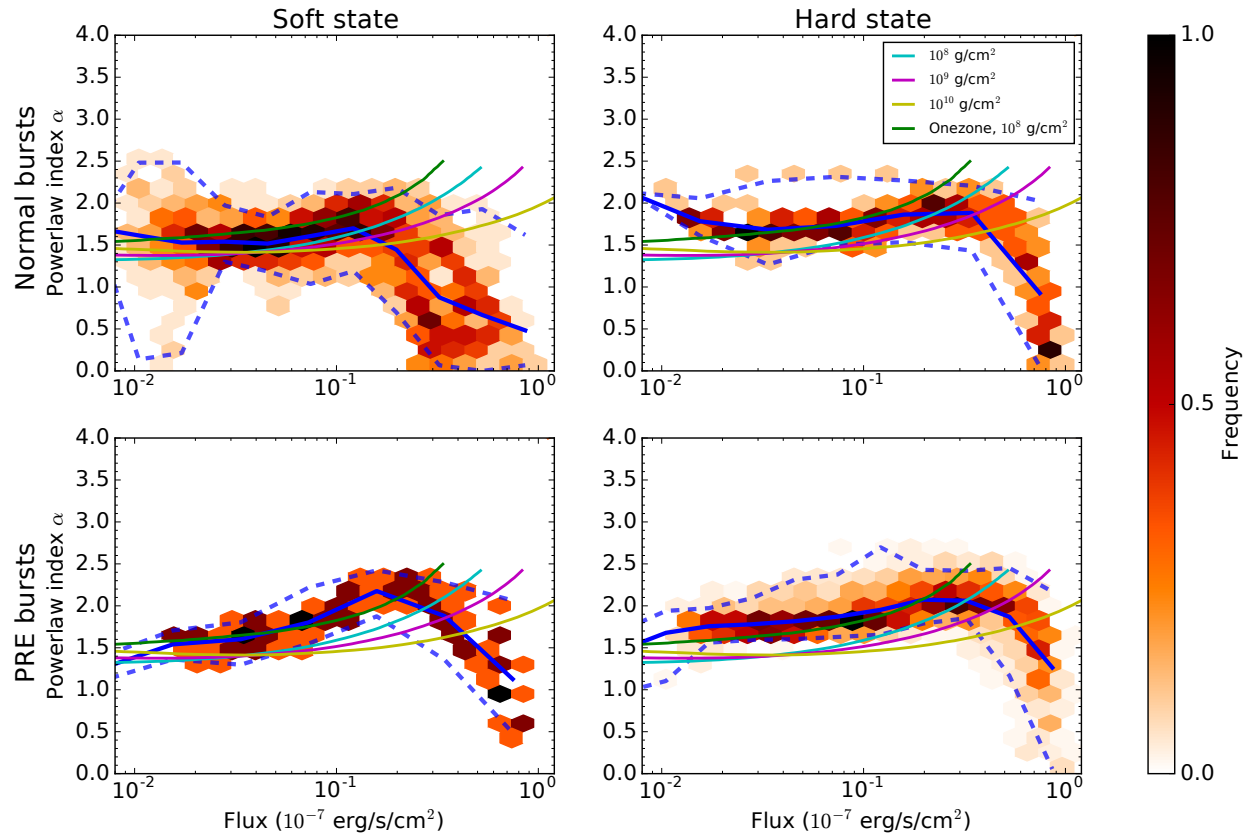

Fig. 5. Same as Fig. 3, but for 4 U 1728-34. The data are shown for PRE bursts (lower panels) and normal bursts (upper panels) in the hard (right panels) and soft state (left
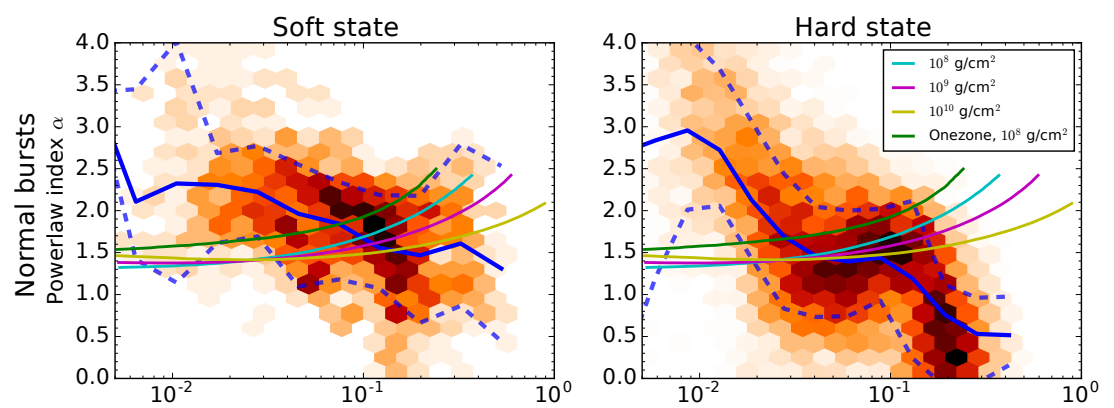
panels).
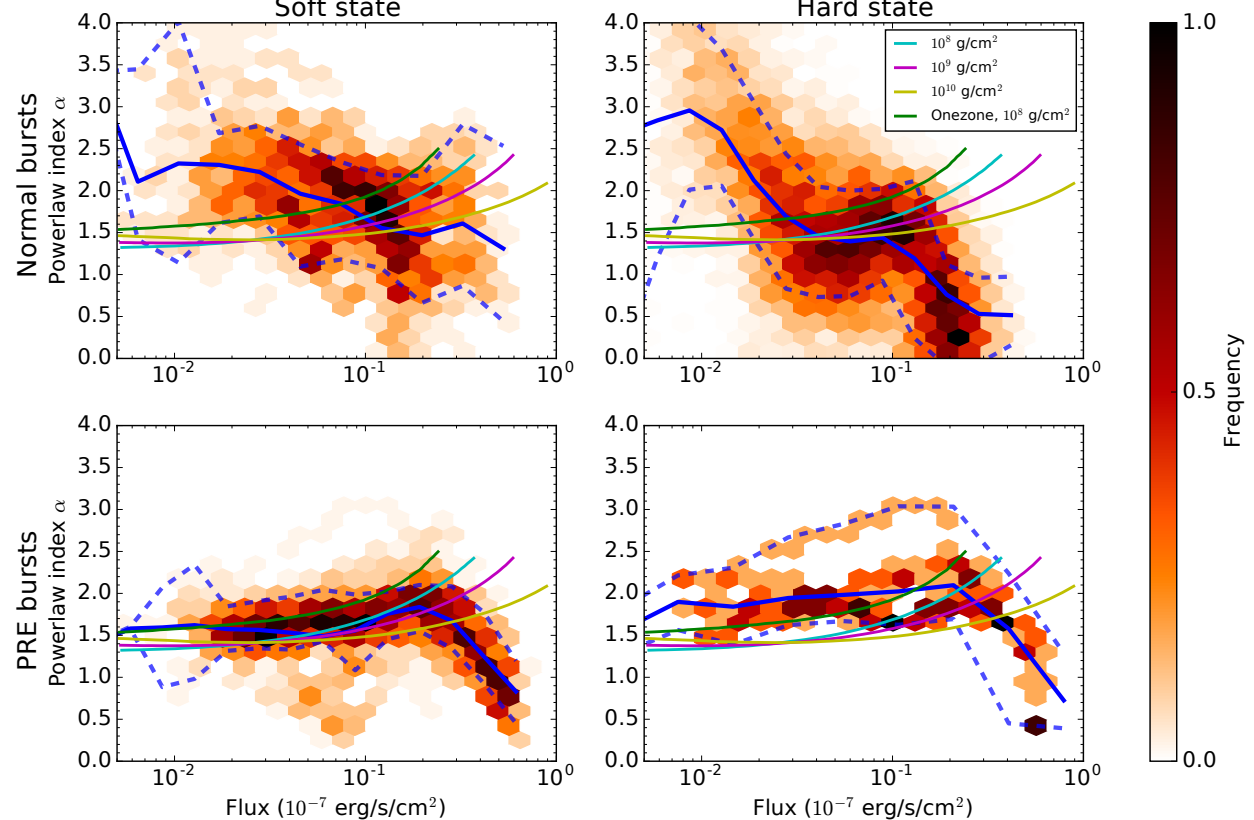

Fig. 6. Same as Fig. 5, but for $4 \mathrm{U} 1636$ 536.

of $\alpha$ at low fluxes resembling the normal bursts in the hard state and also the decay indices show larger scatter than in the soft state PRE bursts.

\section{Discussion}

The type-I X-ray burst decays have been traditionally described with a single exponential function and recently also with a single power law (in't Zand et al. 2014). Our results show that, while the flux decay during some of the X-ray burst cooling tails can indeed be approximated with a power law, clearly not all the bursts can be fitted with just one constant power-law decay index $\alpha$ because the cooling rate changes in time (see Figs. 3-7). This is also predicted by the cooling models of Cumming \& Macbeth (2004).

Burst cooling is more complex than traditionally thought. As one can see from Figs. 3-7, there are differences in the cooling between different sources and burst types; the most notable difference is the rise in the cooling rate at lower fluxes. As the simplest case, we can consider 3A 1820-303, which is known to be an ultra-compact binary accreting mainly helium (Stella et al. 1987). All of the bursts from this source are thought to be produced by unstable burning of helium; we refer to these bursts as helium bursts. These bursts are found to be qualitatively similar to the simple cooling models of Cumming \& Macbeth (2004) and in't Zand et al. (2014). Similarly, 4U 1728-34 is also believed to be an ultra-compact binary (Galloway et al. 2010), and comparing Figs. 3 and 5 certainly supports this notion because the $\alpha$-flux evolution is very similar. All the bursts from 4U 172834 , regardless of the state or burst type, seem to follow the cooling models, even better than the bursts from 3A 1820-303. Comparing these two He-rich sources and the cooling models, we can argue that the helium bursts can be qualitatively described by the cooling models of Cumming \& Macbeth (2004). One reason for this is that these models, initially constructed for superbursts, assume instantaneous injection of energy, which is a reasonable 

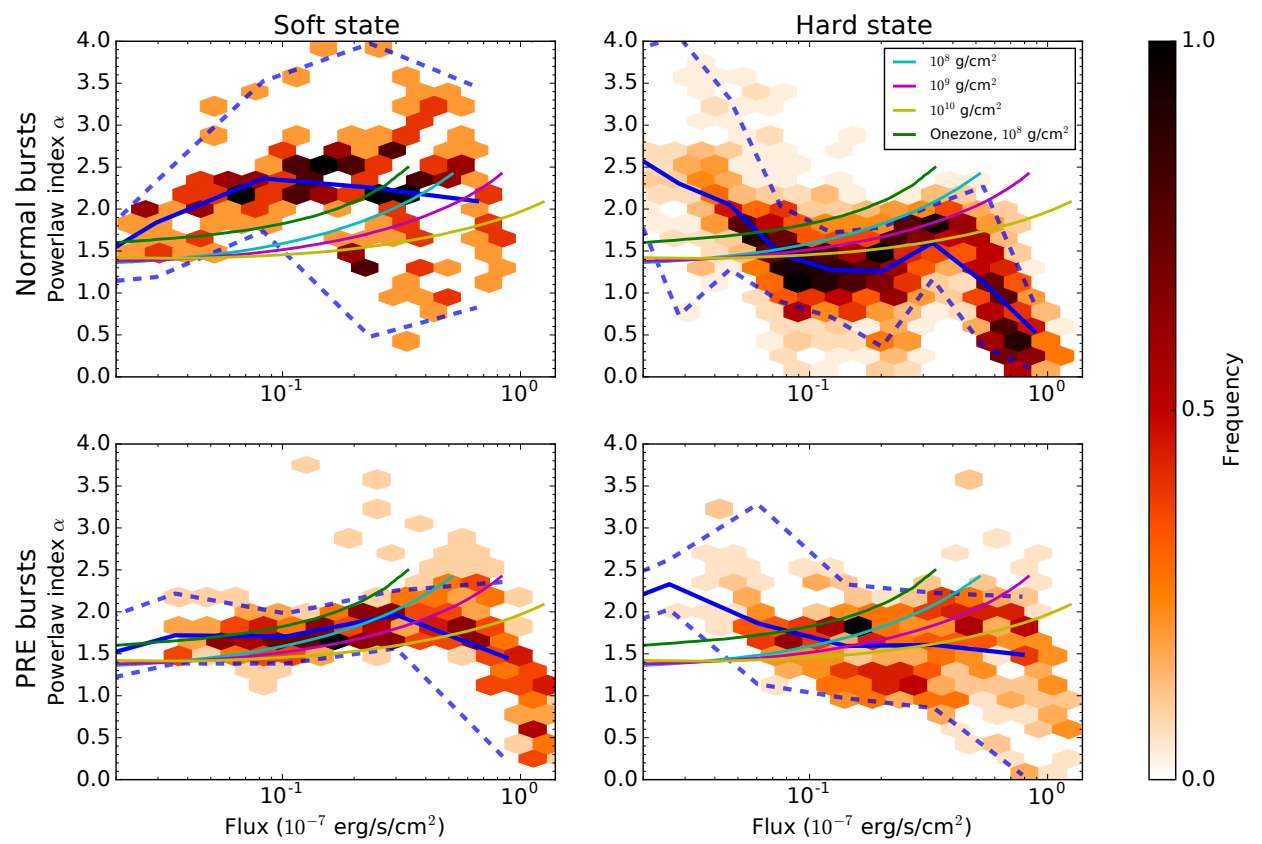

Fig. 7. Same as Fig. 5, but for 4 U 1608-52.

assumption for the helium bursts that rapidly exhaust the available fuel via the fast triple-alpha process.

Hydrogen, on the other hand, burns much slower via $\beta$ decay limited hot CNO-cycle (Fowler \& Hoyle 1965) and rpprocess (Wallace \& Woosley 1981), so the energy is released slower. How all this then affects the evolution of $\alpha$ is most evident in GS 1826-24, which is, in contrast, known to be a H-rich source with prominent rp-burning tails (Galloway et al. 2004; Heger et al. 2007). The cooling of bursts in GS 1826-24 is very different from the He-rich sources. The cooling tail of these bursts can be divided into three phases. First the $\alpha$ stays roughly constant at about 1 , while the hydrogen is still burning via the rp-process (Heger et al. 2007). After that the cooling rate increases almost linearly to $\alpha \approx 4$, meaning that the flux decays exponentially in contrast to the power-law-like cooling of the He-rich sources. The final third phase of the decay at the very end of the burst, where the $\alpha$ seems to decrease, may be the onset of the one-hour long tails observed by in't Zand et al. (2009), which can be explained by delayed cooling of deeper layers that were heated up through inward conduction.

The flux decay behaviour of 4U 1636-536 and 4U 160852 is more complex than that of the previous sources, as there are differences between the accretion states. All the PRE bursts of $4 \mathrm{U} 1636-536$ and the soft state PRE bursts of 4U 1608-52 are similar to the bursts of the two helium sources, and they also follow the simple cooling models. These bursts support the argument of Ebisuzaki \& Nakamura (1988) and Zhang et al. (2011) that PRE bursts are $\mathrm{H}$ poor. On the other hand, the hard state PRE bursts of 4U 1608-52 differ. They are similar to the hard state normal bursts because in both of these the power-law index $\alpha$ rises at lower burst fluxes as normal bursts of 4U 1636-536 do in the hard state. The increase in $\alpha$ is similar to GS 182624 , which is known to be in the "Case III" burning regime of Narayan \& Heyl (2003) producing mixed H/He bursts with stable $\mathrm{H}$ burning in between, but the $\alpha$-evolution is still quantitatively different. In the bursts from GS 1826-24 the $\alpha$ is at first less than one, i.e. there is hardly any cooling at all, possibly because of the rp-burning. In both 4U 1636-536 and 4U 1608-52 the $\alpha$ is higher, around 1.5, indicating that the cooling is faster. The rise is the most prominent in GS 1826-24 and most subtle in $4 \mathrm{U} 1608-52$. This increase in the cooling rate at lower burst fluxes is seen only in these three supposedly H-rich sources. The differences could arise because of the different amount of available hydrogen, variations in metallicity, or in the area on which new fuel accretes (Bildsten 2000).

The situation of the soft state normal bursts of 4U 1636-536 and $4 \mathrm{U} 1608-52$ is more complex. All soft state bursts have been suggested as $\mathrm{H}$ poor (e.g. Zhang et al. 2011), and 4U 1608-52 supports this notion, to some extent, since all the soft state bursts are similar to $3 \mathrm{~A} 1820-303$. In $4 \mathrm{U} 1636-536$ the $\alpha-F$ evolution of the soft state normal bursts seems to be somewhere in between the H-poor PRE and the H-rich hard state normal bursts. This could be partly because in 4U 1636-536 the accretion states are not as well defined as in 4U 1608-52 (see Fig. 1) and we have neglected the intermediate state that can, in fact, be more similar to the soft state than to the hard state.

Our results show that while the helium bursts can be qualitatively described with the simple models of Cumming \& Macbeth (2004), the mixed bursts display more complex behaviour. On the other hand, Heger et al. (2007) showed that the burst light curves of GS 1826-24 can be well reproduced with models of Woosley et al. (2004), which include a large nuclear reaction network to follow the rp-process. But there are also some differences between the models, depending on the nuclear physics and the physical treatment. This is evident in Figs. 3-7 as well, where the one-zone and multi-zone models produce different results (see also Cyburt et al. 2016).

The most comprehensive, and also the most recent, modelling of bursting behaviour has been carried out by Lampe et al. (2016), who used the KEPLER hydrodynamic code to simulate bursts with varying accretion rates across a range of chemical compositions; these simulations also included the He-rich and solar-like $\mathrm{H} / \mathrm{He}$ ratios, which are similar to the sources in our work. A detailed comparison with these models and the data is therefore in order.

Lampe et al. (2016) found that the accretion rate is one of the governing factors of bursting properties and burst morphology. Our results support this conclusion. The accretion state seems to affects the burst cooling, as we observe mixed $\mathrm{H} / \mathrm{He}$ bursts with the rise in the power-law index only in the hard state (low 
persistent flux) and pure He bursts in the soft state (higher persistent flux), which is opposite from what Narayan \& Heyl (2003) have predicted. This may be due to the differences in the accretion geometry between these two states. In the hard state the accretion flow onto the surface is more likely spherical and consists of hot electrons, protons, and heavier ions that deposit most of their energy at the upper atmosphere layers (Bildsten et al. 1992). In the soft state, instead, the flow settles onto the neutron star surface through the optically thick boundary/spreading layer, where the flow spreads from the NS equator to higher latitudes depending on the accretion rate (Inogamov \& Sunyaev 1999, 2010). Furthermore, the settling of matter onto the neutron star is also expected to cause significant heat dissipation in the burning depths, and thus the formation of a spreading layer in the soft state may cause changes in burning regimes.

In the end, we conclude that studying the cooling of the X-ray bursts with the aforementioned dynamic power-law method can be a very powerful tool in probing the interiors of NSs. Firstly, we get indirect information about the fuel composition because $\mathrm{H}$-rich and $\mathrm{H}$-poor bursts produce characteristically different cooling shapes. Secondly, we can study the burst ignition and burning mechanism because the slope of the flux decay seems to be sensitive to any additional heating, such as the prolonged rp-burning in the NS ocean. Lastly, it is interesting to see how the actual physical environment of the NS affects and modifies the cooling. Here the differences in the accretion rate might also play a role because of the clear differences between the bursts occurring in the hard and soft states. As a next step it is also possible to do a similar analysis to simulated bursts such as those produced by the KEPLER code (see Lampe et al. 2016). Hence, when the input is known, we can calibrate our method further and study, for example the effects of composition and accretion in more detail. This is a matter of our follow-up work.

Acknowledgements. We thank Jean in 't Zand and Duncan Galloway for interesting discussions. J.K., J.J.E.K., and J.P. acknowledge support from the Academy of Finland grant 268740. J.J.E.K. also acknowledges support from the ESA research fellowship programme and the Academy of Finland grant 295114. J.N. acknowledges support from the University of Turku Graduate School in Physical and Chemical Sciences. J.P. was partly supported by the Foundations' Professor Pool, the Finnish Cultural Foundation, and the National Science Foundation grant PHY-1125915.

\section{References}

Asai, K., Dotani, T., Nagase, F., \& Mitsuda, K. 2000, ApJS, 131, 571

Augusteijn, T., van der Hooft, F., de Jong, J. A., van Kerkwijk, M. H., \& van Paradijs, J. 1998, A\&A, 332, 561

Babushkina, O. P., Kudriavtsev, M. I., Melioranskii, A. S., et al. 1975, Sov. Astron. Lett., 1, 32

Belian, R. D., Conner, J. P., \& Evans, W. D. 1972, ApJ, 171, L87

Bildsten, L. 1998, in NATO Advanced Science Institutes (ASI) Series C, eds R. Buccheri, J. van Paradijs, \& A. Alpar, 515, 419
Bildsten, L. 2000, in AIP Conf. Proc., eds. S. S. Holt \& W. W. Zhang, 522, 359 Bildsten, L., Salpeter, E. E., \& Wasserman, I. 1992, ApJ, 384, 143

Chenevez, J., Galloway, D. K., in 't Zand, J. J. M., et al. 2016, ApJ, 818, 135 Cumming, A., \& Macbeth, J. 2004, ApJ, 603, L37

Cyburt, R. H., Amthor, A. M., Heger, A., et al. 2016, ApJ, 830, 55

D’Aí, A., di Salvo, T., Iaria, R., et al. 2006, A\&A, 448, 817

Done, C., Gierliński, M., \& Kubota, A. 2007, A\&ARv, 15, 1

Ebisuzaki, T., \& Nakamura, N. 1988, ApJ, 328, 251

Fowler, W. A., \& Hoyle, F. 1965, Nucleosynthesis in Massive Stars and Supernovae (Chicago: University Chicago Press)

Galloway, D. K., Cumming, A., Kuulkers, E., et al. 2004, ApJ, 601, 466

Galloway, D. K., Psaltis, D., Muno, M. P., \& Chakrabarty, D. 2006, ApJ, 639, 1033

Galloway, D. K., Muno, M. P., Hartman, J. M., Psaltis, D., \& Chakrabarty, D. 2008, ApJS, 179, 360

Galloway, D. K., Yao, Y., Marshall, H., Misanovic, Z., \& Weinberg, N. 2010, ApJ, 724, 417

García, F., Zhang, G., \& Méndez, M. 2013, MNRAS, 429, 3266

Giles, A. B., Hill, K. M., Strohmayer, T. E., \& Cummings, N. 2002, ApJ, 568, 279

Grindlay, J., \& Gursky, H. 1976, ApJ, 205, L131

Grindlay, J., Gursky, H., Schnopper, H., et al. 1976, ApJ, 205, L127

Hasinger, G., \& van der Klis, M. 1989, A\&A, 225, 79

Heasley, J. N., Janes, K. A., Zinn, R., et al. 2000, AJ, 120, 879

Heger, A., Cumming, A., Galloway, D. K., \& Woosley, S. E. 2007, ApJ, 671, L141

Inogamov, N. A., \& Sunyaev, R. A. 1999, Astron. Lett., 25, 269

Inogamov, N. A., \& Sunyaev, R. A. 2010, Astron. Lett., 36, 848

in't Zand, J. J. M., Heise, J., Kuulkers, E., et al. 1999, A\&A, 347, 891

in't Zand, J. J. M., Keek, L., Cumming, A., et al. 2009, A\&A, 497, 469

in't Zand, J. J. M., Cumming, A., Triemstra, T. L., Mateijsen, R. A. D. A., \& Bagnoli, T. 2014, A\&A, 562, A16

Jonker, P. G., \& Nelemans, G. 2004, MNRAS, 354, 355

Kajava, J. J. E., Nättilä, J., Latvala, O.-M., et al. 2014, MNRAS, 445, 4218

Keek, L., in't Zand, J. J. M., Kuulkers, E., et al. 2008, A\&A, 479, 177

Keek, L., Cumming, A., Wolf, Z., et al. 2015, MNRAS, 454, 3559

Koljonen, K. I. I., Kajava, J. J. E., \& Kuulkers, E. 2016, ApJ, 829, 91

Kuulkers, E., Homan, J., van der Klis, M., Lewin, W. H. G., \& Méndez, M. 2002, A\&A, 382, 947

Kuulkers, E., den Hartog, P. R., in't Zand, J. J. M., et al. 2003, A\&A, 399, 663

Lampe, N., Heger, A., \& Galloway, D. K. 2016, ApJ, 819, 46

Lewin, W. H. G., van Paradijs, J., \& Taam, R. E. 1993, Space Sci. Rev., 62, 223

Muñoz-Darias, T., Motta, S., \& Belloni, T. M. 2011, MNRAS, 410, 679

Muñoz-Darias, T., Fender, R. P., Motta, S. E., \& Belloni, T. M. 2014, MNRAS, 443, 3270

Narayan, R., \& Heyl, J. S. 2003, ApJ, 599, 419

Poutanen, J., Nättilä, J., Kajava, J. J. E., et al. 2014, MNRAS, 442, 3777

Rappaport, S., Ma, C. P., Joss, P. C., \& Nelson, L. A. 1987, ApJ, 322, 842

Stella, L., Priedhorsky, W., \& White, N. E. 1987, ApJ, 312, L17

Strohmayer, T., \& Bildsten, L. 2006, in Compact Stellaer X-ray Sources, eds.

W. H. G. Lewin, \& M. van der Klis (Cambridge University Press), 113

Suleimanov, V., \& Poutanen, J. 2006, MNRAS, 369, 2036

Sztajno, M., van Paradijs, J., Lewin, W. H. G., et al. 1986, MNRAS, 222, 499

Ubertini, P., Bazzano, A., Cocchi, M., et al. 1999, ApJ, 514, L27

van Paradijs, J., \& Lewin, H. G. 1986, A\&A, 157, L10

van Paradijs, J., van der Klis, M., van Amerongen, S., et al. 1990, A\&A, 234, 181

Wachter, S., Hoard, D. W., Bailyn, C. D., Corbel, S., \& Kaaret, P. 2002, ApJ, 568,901

Wallace, R. K., \& Woosley, S. E. 1981, ApJS, 45, 389

Woosley, S. E., Heger, A., Cumming, A., et al. 2004, ApJS, 151, 75

Worpel, H., Galloway, D. K., \& Price, D. J. 2013, ApJ, 772, 94

Zhang, G., Méndez, M., \& Altamirano, D. 2011, MNRAS, 413, 1913 